\title{
SYSTEMIC SCLEROSIS WITH SEVERE AND RAPIDLY PROGRESSIVE MYOCARDIAL FIBROSIS: A CASE REPORT
}

Caio Carvalho Inacio de Vasconcellos ${ }^{1}$,, , Maria Cecilia da Fonseca Salgado ${ }^{1}$, Ana Paula Casseta Nucera ${ }^{1}$, Alessandra Cardoso

Pereira', Camila Souto', Felipe Cesar Freire', João Luiz Pereira Vaz'

1.Universidade Federal do Estado do Rio de Janeiro, Rio de Janeiro (RJ), Brazil.

*Corresponding author: caiocdevasconcellos@gmail.com

\section{BACKGROUND}

Primary cardiac involvement in systemic sclerosis has uncertain prevalence due to the heterogeneity of manifestations and the variability of the techniques used for its diagnosis. The objective of this report is to present a case of systemic sclerosis with mainly cardiac involvement, severe and rapidly progressing, and to highlight the need to identify predictors of the development of severe primary cardiac injury.

\section{CASE REPORT}

Female patient begins to present Raynaud's phenomenon at 22 years old. In 2009, at the age of 26, she started following up with a rheumatologist. She presented nuclear fine speckled ANA (1:80), positive anti-scl-70 antibody and cutaneous involvement on the face, hands and forearms, being diagnosed with systemic sclerosis. She also had significant muscular involvement in the shoulder girdle, with difficulty in abducting the upper limbs and great muscular atrophy, with EMG and biopsy compatible with myopathy, but the enzymes were normal. Until 2014, the case evolved well, controlled with methotrexate and penicillamine. A routine Doppler echocardiogram in 2014 did not show any alterations. In 2015, she moved abroad and had a full-term pregnancy in 2016. After childbirth, the condition worsened and she evolved with lower limb edema and Raynaud. She used mycophenolate in a dose of up to $2 \mathrm{~g}$ for more than six months, later removed due to important diarrhea. Between January and February 2019, she was hospitalized for a sudden onset anasarca and significant dyspnea, compatible with decompensated congestive heart failure. The echocardiogram and magnetic resonance imaging were compatible with severe myocardial fibrosis. Corticosteroid pulse therapy was performed where she lived. Back to Brazil in May 2019, drugs were prescribed to control the heart failure and the rheumatological disease, with poor response. After adjusting the dose of betablockers and digoxin, she presented generalized edema and cyanosis of extremities and face and developed jugular and subclavian thrombosis, without response to anticoagulation. Three months later, a Rituximab cycle was performed. After one month, the patient had community-acquired pneumonia and severe pericarditis. Admitted to a private hospital, she progressed to worsening of the pulmonary condition, sepsis and death.

\section{CONCLUSION}

We highlight the need of studies to identify clinical and laboratory factors with a potential predictor of the development of heart disease. Although there is still no specific therapy, the development of an evidence-based screening routine can allow the early management of these cases. 